\title{
Regresión lineal de mínimos cuadrados para la carga y descarga de un condensador
}

\section{Linear regression of least squares for the charge and discharge of capacitor}

\author{
Libardo Santiago' ${ }^{1}$, Olga Lucy Rincón-Leal ${ }^{2}$
}

Forma de citar: L. Santiago, O. L. Rincón, Regresión lineal de mínimos cuadrados para la carga y descarga de un condensador, Eco.Mat. 2015; 6(1): 34-42.

Recibido:

Marzo 20 de 2015

Aceptado:

Mayo 25 de 2015

\section{RESUMEN}

El objetivo es determinar experimentalmente el proceso de carga y descarga de un condensador estando conectado en serie con una resistencia y una fuente de corriente continua, utilizando para ello los métodos numéricos en el proceso de ajuste de curvas a través de la regresión lineal de mínimos cuadrados, y con ayuda del software matemático Matlab se realizó la construcción de las respectivas curvas.

Se estableció una relación entre el voltaje y el tiempo, formándose así una gráfica exponencial; se dedujeron las relaciones existentes y el comportamiento del fenómeno, dadas las ecuaciones y con la ayuda de la segunda Ley de Kirchhoff; se determinaron las contantes de tiempo utilizando el método de regresión lineal de mínimos cuadrados, encontrándose un error experimental del $5 \%$ con respecto a la constate de tiempo teórica RC.

Palabras Claves: Condensador, resistencia y regresión lineal.

\section{ABSTRACT}

The objective is to determine experimentally S. Process Loading and unloading of un capacitor being connected in series with a resistor and a current source, using para This numerical methods in the process of adjustment curves Through linear regression Least Squares, with the help of mathematical software Matlab building the respective curves was performed.

A relationship between the voltage and time, thus forming exponential graph $\mathrm{A}$ is established; the relationships and the behavior of the phenomenon were deducted, given the equations and with the help of the Second Law of Kirchhoff; the Time constants were determined using the method of least squares linear regression, experimental errors UN meeting 5\% with respect to the I Theoretical RC time constant.

Keywords: Condenser, resistance and lineal regression.
${ }^{1}$ Magister en Física. Universidad Francisco de Paula Santander. libardsan@hotmail.com

${ }^{2}$ Magister en Educación Matemática. Universidad Francisco de Paula Santander. olgarincon@ufps.edu.co 


\section{INTRODUCCIÓN}

El condensador es un dispositivo comúnmente utilizado para almacenar energía eléctrica. Para poder almacenar energía en un dispositivo como este se genera una diferencia de potencial entre dos materiales conductores los cuales se separan por un material dieléctrico.

Cuando se conecta un condensador a una fuente de voltaje, los electrones (cargas) tratan de moverse de un extremo de la fuente al otro. Pero como hay un material dieléctrico en el camino, no pueden pasar y se acumulan en los conductores. Este proceso proporciona cargas iguales y de signo contrario a los conductores. Si se desconecta la fuente de voltaje, las cargas que se habían acumulado no encontrarán un camino que les permita abandonar a los conductores, por lo que quedarán atrapadas en el dispositivo el cual queda cargado y almacena una energía. La energía que puede almacenar un condensador depende de la cantidad de cargas que se acumulen en los conductores. Obviamente, esto depende del voltaje proporcionado por la fuente, pero también depende de la geometría de los conductores y de las características del dieléctrico. [1]

Para cuantificar las características de almacenamiento de energía de un capacitor, se le asocia un número llamado: capacitancia.

Se define la capacitancia $\mathrm{C}$ de un condensador como la razón de la magnitud de la carga en cualquiera de los dos conductores y la diferencia de potencial entre ellos.

Un Faraday es la capacitancia C de un conductor que retiene un coulomb de carga por cada volt de potencial.

$$
C=\frac{Q}{V}\left[\frac{C}{V}\right] ; \quad \text { Faraday }=\frac{\operatorname{coulomb}(c)}{\operatorname{volt}(V)}
$$

El capacitor es un dispositivo que almacena energía en un campo electrostático. Una lámpara de destello requiere de una emisión breve de energía eléctrica, otros capacitores más grandes se emplean para proveer intensas pulsaciones de láser con el fin de inducir una fusión termonuclear en pequeñas bolitas de hidrogeno. [2]

Las leyes de Kirchhoff tienen como finalidad la obtención de un sistema de ecuaciones cuya resolución, por métodos matemáticos, nos permita conocer las intensidades de corriente en sentido y valor existentes en un circuito. Estas son:

1) Ley de los nudos: la suma algebraica de las intensidades en un nudo es cero $\Sigma \mathrm{I}_{\mathrm{i}}=0$

2) Regla de las mallas: la suma algebraica de las fuerzas electromotrices aplicadas a una malla es igual a la suma de las caídas de tensión de dicha malla.

$$
\Sigma \mathrm{E}_{i}=\Sigma \mathrm{I}_{i} * \mathrm{R}_{i}
$$

\section{DESARROLLO DE LA EXPERIENCIA}

Para comenzar con el experimento pasaremos a analizar primero el fenómeno de carga y descarga de un condensador en un circuito $\mathrm{RC}$, conformado en este caso por una resistencia y un condensador conectados en serie a través de una fuente de alimentación con un voltaje terminal igual a la FEM de la batería (figura 1), despreciando la resistencia interna de la misma, para poder simplificar el análisis presentado a continuación. [4]

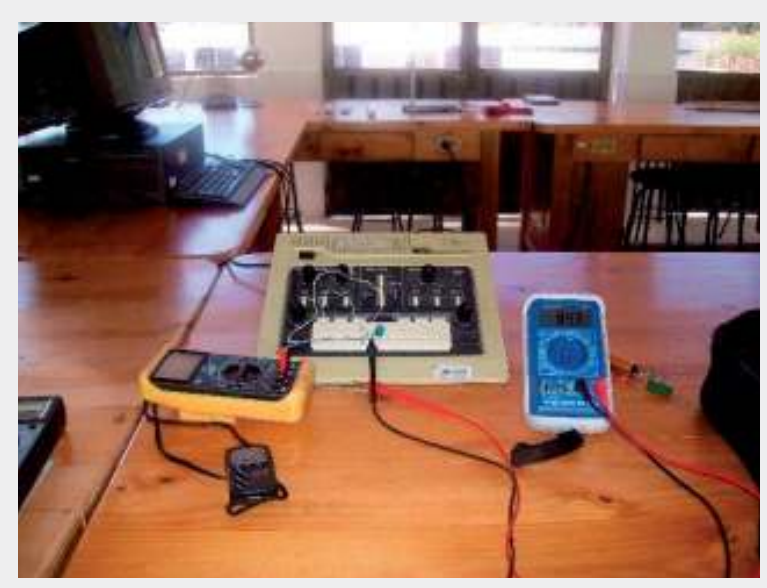

Figura 1. Montaje de circuito RC para el estudio de carga y Descarga de un condensador 
Eco.Mat

Cúcuta-Colombia

Vol. 6

No. 1

Enero-Diciembre 2015

ISSN 1794-8231

ISSN 2462 - $8794 \mathrm{Wel}$

PP: $34-42$ de este circuito para comenzar con el análisis.

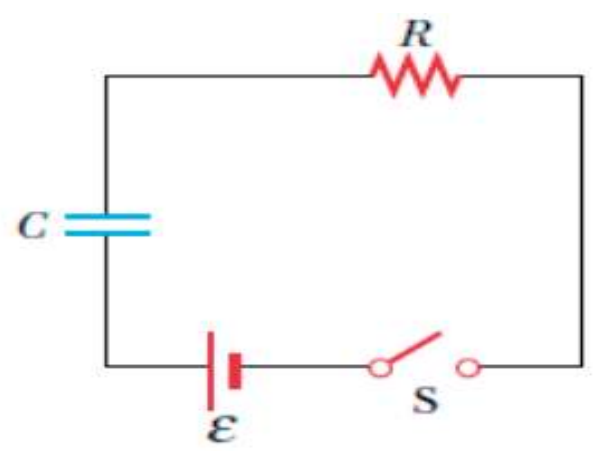

Figura 2. Circuito RC para el proceso de Carga del condensador.

Se parte de un sistema en el cual el condensador está inicialmente descargado, como el interruptor no cierra el circuito no existe corriente alguna en el sistema por lo que si definimos $\mathrm{t}=0$ al cerrar el interruptor, la carga comenzará a recorrer por el circuito estableciendo una corriente en el mismo y el capacitor empezará a cargarse, que terminará cuando este se encuentre a la misma diferencia de potencial que la fuente. [5]

De acuerdo al proceso matemático de carga que varía en el tiempo y en el cual la resistencia $\mathrm{R}$ influye, aplicándolo a la segunda ley de Kirchhoff o ley de las mallas, el cual define lo siguiente:

$$
\sum_{\substack{\text { Circuito } \\ \text { cerrado }}} \Delta V=0
$$

El cual señala que la suma de las diferencias de potencial aplicadas a todos los elementos que conforman un circuito cerrado debe ser igual a 0 .

Mediante esta ley obtenemos lo siguiente:

$$
E-\frac{q}{C}-I R=0
$$

Donde $E$ es la diferencia de potencial de la fuente. $\frac{q}{C}$ la diferencia de potencial del condensador y $I R$ la diferencia de potencial de la resistencia. Para la determinación de los signos utilizamos la manera convencional. La asignación de la dirección de la corriente, el cual señala que esta tiene la misma dirección que el flujo de la carga positiva.

Definimos los valores máximos tanto de la corriente como de la carga en el sistema. En $t=0$ como mencionábamos la $\Delta V$ en el condensador es igual a 0 por lo que la ecuación de la segunda ley de Kirchhoff nos queda: [5]

$$
\begin{aligned}
& E-I R=0 \\
& I_{\text {máx }}=\frac{E}{R}
\end{aligned}
$$

Es decir en el estado inicial, la diferencia de potencial presente en el resistor es la misma que en la fuente y por lo tanto la corriente presente en este estado del circuito en máxima.

Ahora en el otro extremo cuando $t \rightarrow \infty$ la diferencia de potencial presente en el capacitor será la misma que en la fuente y por lo tanto al no existir corriente la diferencia de potencial aplicada al resistor resulta ser 0 y entonces la segunda ley de Kirchhoff nos queda:

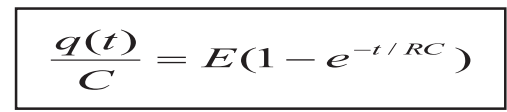

$$
\begin{aligned}
& E-\frac{q}{C}=0 \\
& Q_{\text {máx }}=E C
\end{aligned}
$$

Es decir la diferencia de potencial aplicada en el condensador es la misma que la de la fuente y por ende la carga del mismo es máxima. [5]

Utilizando la Ec (3) y que $I=d q / d t$. Hallemos la expresión matemática para el proceso de carga del condensador. 
En $\mathrm{t}=0$ el voltaje de $\operatorname{La} \operatorname{Ec}(8)$ es $V(0)=E$; el $E-\frac{q}{C}-\frac{d q}{d t} R=0$

$$
\frac{d q}{d t}=\frac{C E}{R C}-\frac{q}{R C}
$$$$
\frac{d q}{d t}=-\frac{q-C E}{R C}
$$$$
\int_{0}^{q} \frac{d q}{q-C E}=-\int_{0}^{t} \frac{d t}{R C}
$$

$$
\operatorname{Ln}\left(\frac{C E-q}{C E}\right)=-\frac{t}{R C}
$$

$$
\begin{aligned}
& \frac{C E-q}{C E}=e^{-t / R C} \\
& q(t)=C E\left(1-e^{-t / R C}\right)
\end{aligned}
$$

Podemos expresar la carga como el voltaje aplicado al capacitor en función del tiempo de la siguiente manera: [6]

$$
\mathrm{V}(\mathrm{t})_{\text {capacitor }}=\mathrm{E}\left(1-\mathrm{e}^{-\mathrm{t} / \mathrm{RC}}\right)
$$

Para hallar la corriente del sistema en tenemos que derivar la carga respecto del tiempo.

$$
\begin{aligned}
& \frac{d q}{d t}=\frac{d\left[C E\left(1-e^{-t / R C}\right)\right]}{d t} \\
& I(t)=\frac{E}{R}\left(e^{-t / R C}\right)
\end{aligned}
$$

También podemos expresar la corriente como el voltaje aplicado a la resistencia en función del tiempo de la siguiente manera:

$$
\begin{aligned}
& R I(t)=E\left(e^{-t / R C}\right) \\
& V(t)_{\text {resistor }}=E\left(e^{-t / R C}\right)
\end{aligned}
$$

mismo de la fuente y el voltaje de la $\operatorname{Ec}(6)$ es $V(0)=0$; de lo que podemos deducir:

\section{DESCARGA DE UN CONDENSADOR}

Una vez terminado el proceso de carga del condensador pasaremos a analizar la descarga del mismo en función del tiempo, para esto imaginemos que el condensador del circuito de la figura 3 (cuyo interruptor debe cerrar el circuito) ahora está totalmente cargado y no existe corriente alguna en el sistema, entonces pasaremos a abrir el interruptor y extraer del

[5]

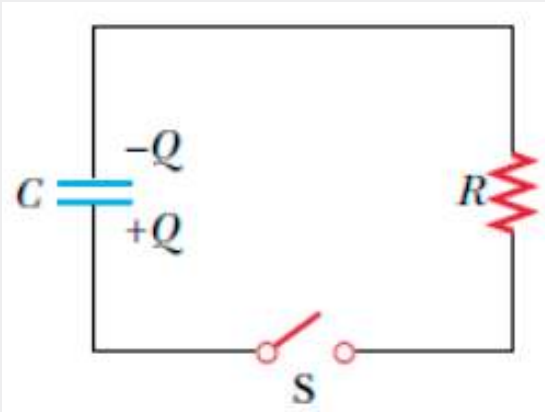

Figura 3. Circuito RC para el proceso de Descarga del condensador.

Una vez que el circuito se encuentre cerrado por el interruptor comenzar a fluir corriente desde la placa positiva inferior del capacitor hacia la placa superior negativa del mismo, descargándose, pero a la vez esta corriente

$$
V(t)_{\text {resistencia }}=E-V(t)_{\text {condensador }}
$$
sistema la fuente de alimentación.

a través del resistencia por lo cual nuestra malla para este sistema es:

$$
\begin{aligned}
& -\frac{q}{C}-R I=0 \\
& -\frac{q}{C}-R \frac{d q}{d t}=0
\end{aligned}
$$


Eco.Mat

Cúcuta-Colombia

Vol. 6

No. 1

Enero-Diciembre 2015 ISSN 1794-8231

ISSN 2462 - 8794 Web PP: $34-42$

$\int_{\text {Qnáx }}^{q} \frac{d q}{q}=-\int_{o}^{t} \frac{d t}{R C}$

$\operatorname{Ln}\left(\frac{q}{Q m a ́ x}\right)=-\frac{t}{R C}$

$\frac{q}{Q m a ́ x}=e^{-t / R C}$

$q(t)=(Q m a ́ x) e^{-t / R C}$.

Expresar la carga $q(t)$ como el voltaje aplicado al capacitor en función del tiempo de la siguiente manera: [6]

$Q(t)=C E e^{-t / R C}$

$\frac{q(t)}{C}=E e^{-t / R C}$

$V(t)_{\text {capacitor }}=E e^{-t / R C}$

Para hallar la corriente del sistema tenemos que derivar la carga respecto del tiempo:

$$
\begin{aligned}
& \frac{d q}{d t}=\frac{d\left(C E e^{-t / R C}\right)}{d t} \\
& I(t)=-\frac{E}{R} e^{-t / R C}
\end{aligned}
$$

El signo menos en esta expresión no indica que la corriente es negativa, lo cual no existe, sino que la dirección de la corriente es inversa a la del proceso de carga del condensador.

Ahora podemos relacionar el voltaje del condensador de la Ec.(11) con la Ec.(12)

$V(t)_{\text {resisrencia }}=-V(t)_{\text {condensador }}$

\section{CONSTANTE DE TIEMPO}

Podemos observar que en todas las expresiones hasta ahora desarrolladas se encuentra un término muy particular $e^{-t /} \boldsymbol{R C}$, el cual nos indica que si el tiempo llegará a ser igual a RC los procesos de carga y descarga tendrían el $\mathbf{e}^{\mathbf{- 1}}(\mathbf{3 6 . 7 9 \%})$ del valor máximo de la corriente máxima en el caso de la carga del capacitor y de la carga máxima, la corriente máxima en el caso de la descarga del capacitor y el $1-\mathbf{e}^{-1}(\mathbf{6 3 . 2 1 \% )}$ del valor máximo de la carga máxima del capacitor en el proceso de carga del mismo. A este término RC que acabamos de mencionar y utilizar para este análisis, se le conoce como la constante de tiempo. [7]

\section{MATERIALES}

- Fuente

- Multímetro digital

- Cronómetro

- Condensador $(\mathrm{C}=3300 \mu \mathrm{F})$

- $\operatorname{Resistencia}(\mathrm{R}=4,86 \mathrm{k} \Omega)$

\section{DATOS OBTENIDOS}

\section{CARGA DEL CONDENSADOR}

TABLA 1. DATOS EXPERIMENTALES DEL VOLTAJE Y EL TIEMPO PARA EL PROCESO DE CARGA DEL CONDENSADOR.

\begin{tabular}{|c|c|c|c|}
\hline $\begin{array}{c}\mathbf{N}^{\mathbf{0}} \mathbf{d e} \\
\text { toma de } \\
\text { datos }\end{array}$ & $\begin{array}{c}\text { Tiempo } \\
\text { (s) }\end{array}$ & $\begin{array}{c}\text { Voltaje } \\
\text { Condensador } \\
(\mathbf{V})\end{array}$ & $\begin{array}{c}\text { Voltaje } \\
\text { Resistencia } \\
(\mathbf{V})\end{array}$ \\
\hline 1 & 0.00 & 0,02 & 12 \\
2 & 7,50 & 4,41 & 7,80 \\
3 & 15,40 & 7,51 & 4,72 \\
4 & 22,61 & 9,20 & 3,00 \\
5 & 31,02 & 10,51 & 1,72 \\
6 & 39,20 & 11,05 & 1,10 \\
7 & 45,81 & 11,30 & 0,87 \\
8 & 54,22 & 11,71 & 0,46 \\
9 & 61,00 & 11,8 & 0,35 \\
10 & 70,20 & 12,00 & 0,12 \\
11 & 76,10 & 12,1 & 0,06 \\
& & & \\
\hline
\end{tabular}

Ahora pasaremos a realizar el análisis de los datos obtenidos en el laboratorio tanto para carga como para la descarga del capacitor. Utilizando los datos de la tabla 1 pasaremos a graficar el voltaje del condensador y de la resistencia versus el tiempo durante el proceso de carga del mismo. [8] 


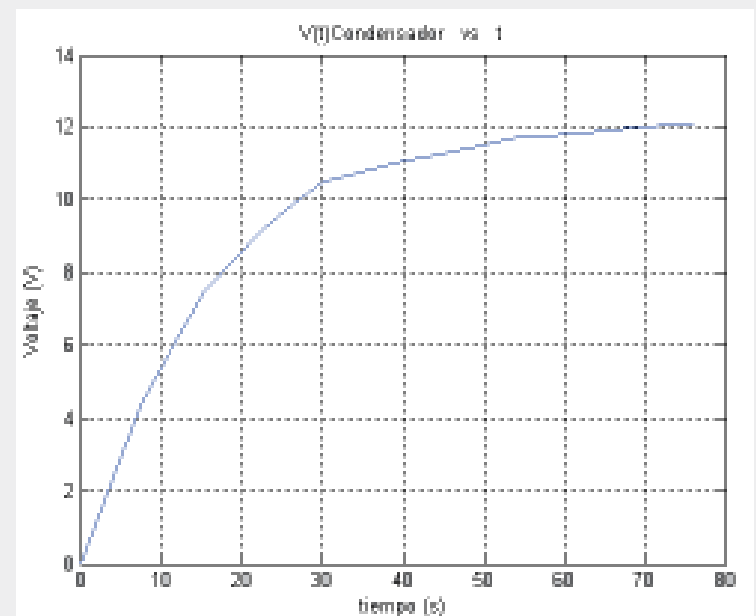

Figura 4. Voltaje del capacitor durante el proceso de Carga en el tiempo

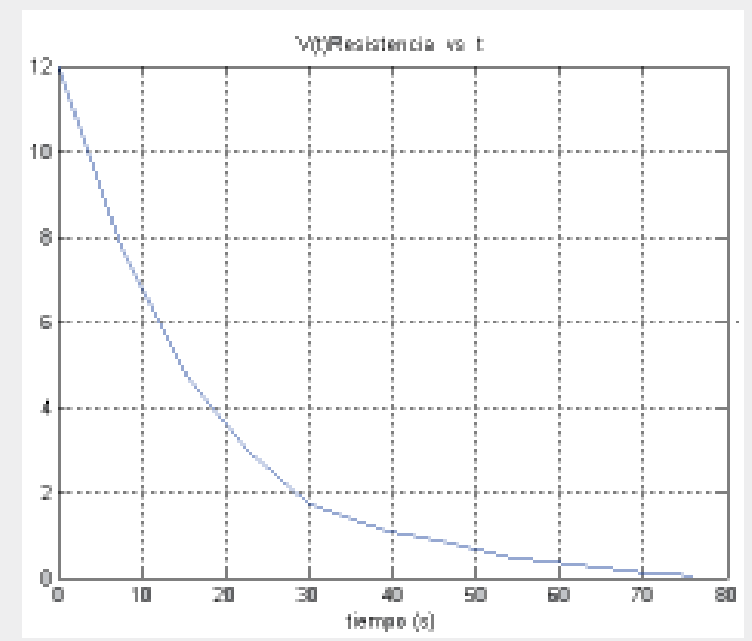

Figura 5. Voltaje de la resistencia durante el proceso de carga en el tiempo.

\section{OBTENCIÓN DE LA CONSTANTE DE TIEMPO $\tau$}

Usemos la Regresión lineal para la obtención de $\mathrm{RC}_{\text {experimental }}$ en el proceso de carga del capacitor usemos la Ec.(6) [9].

$$
V(t)_{\text {capacitor }}=E\left(1-e^{-t / R C}\right)
$$

$E \%=\left[\left(C R_{\text {exp }}-C R_{\text {teórico }}\right) / C R_{\text {teórico }}\right] \times 100$ (16)

$$
\begin{aligned}
& E e^{-t / R C}=E-V(t)_{\text {capacitor }} \\
& \operatorname{Ln}\left(E e^{-t / R C}\right)=\operatorname{Ln}\left(E-V(t)_{\text {capacitor }}\right) \\
& \operatorname{Ln}(E)-\left(\frac{1}{R C}\right) t=\operatorname{Ln}\left(E-V(t)_{\text {capacitor }}\right) \\
& m t=\operatorname{Ln}\left(\frac{E}{E-V(t)_{\text {capacitor }}}\right) \\
& m=\frac{1}{R C}
\end{aligned}
$$

Enero-Diciembre 201 ISSN 1794-8231 ISSN 2462 - $8794 \mathrm{Web}$ PP: $34-42$

El error expérimental asociado a las constantes de tiempo se puede calcular con la expresión :

Calculemos ahora el $\mathrm{RC}_{\text {experimental }}$, para lo cual tenemos que reajustar los datos de la tabla 1

\begin{tabular}{|c|c|c|c|c|c|c|}
\hline $\begin{array}{c}\mathrm{N}^{\mathrm{o}} \text { de } \\
\text { toma } \\
\text { de } \\
\text { datos }\end{array}$ & $\begin{array}{l}\text { Voltaje } \\
\text { (V) }\end{array}$ & $\begin{array}{c}\text { Tiempo } \\
\text { (s) }\end{array}$ & $\begin{array}{c}\text { FUENTE } \\
\text { E(V) }\end{array}$ & $\begin{array}{c}\operatorname{Ln}(E-V i) \\
(\mathrm{V})\end{array}$ & $\begin{array}{c}\operatorname{Ln}(E) \\
\text { (V) }\end{array}$ & $\begin{array}{l}\operatorname{Ln}(\mathbf{E})-\mathbf{L n}\left(\mathrm{E}-V_{i}\right) \\
(\text { adimensional) }\end{array}$ \\
\hline 1 & 0,02 & 0.00 & 12.15 & 2.49 & 2.49 & 0.0016 \\
\hline 2 & 4,41 & 7,51 & 12.15 & 2.05 & 2.49 & 0.45090 \\
\hline 3 & 7,51 & 15,40 & 12.15 & 1.53 & 2.49 & 0.9626 \\
\hline 4 & 9,20 & 22,60 & 12.15 & 1.08 & 2.49 & 1.4155 \\
\hline 5 & 10,51 & 31,02 & 12.15 & 0.49 & 2.49 & 2.0026 \\
\hline 6 & 11,05 & 39,20 & 12.15 & 0.10 & 2.49 . & 2.4020 \\
\hline 7 & 11,30 & 45,81 & 12.15 & -0.16 & 2.49 & 2.6598 \\
\hline 8 & 11,71 & 54,22 & 12.15 & -0.82 & 2.49 & 3.3183 \\
\hline 9 & 11,8 & 61,00 & 12.15 & -1.05 & 2.49 & 3.5472 \\
\hline 10 & 12,00 & 70,20 & 12.15 & -1.90 & 2.49 & 4.3944 \\
\hline
\end{tabular}
para poder utilizar la regresión lineal definida mediante la ecuación (14).

TABLA II. DATOS EXPERIMENTALES DEL VOLTAJE Y EL TIEMPO PARA EL PROCESO DE CARGA DEL CONDENSADOR

Pasemos a graficar los datos de la última columna en función del tiempo para poder hallar la pendiente de la recta determinada por la Ec(14) la cual es el inverso del RC que estamos buscando. 
Eco.Mat

Cúcuta-Colombia

Vol. 6

No. 1

Enero-Diciembre 2015 ISSN 1794-8231

ISSN $2462-8794 \mathrm{Web}$ PP: $34-42$

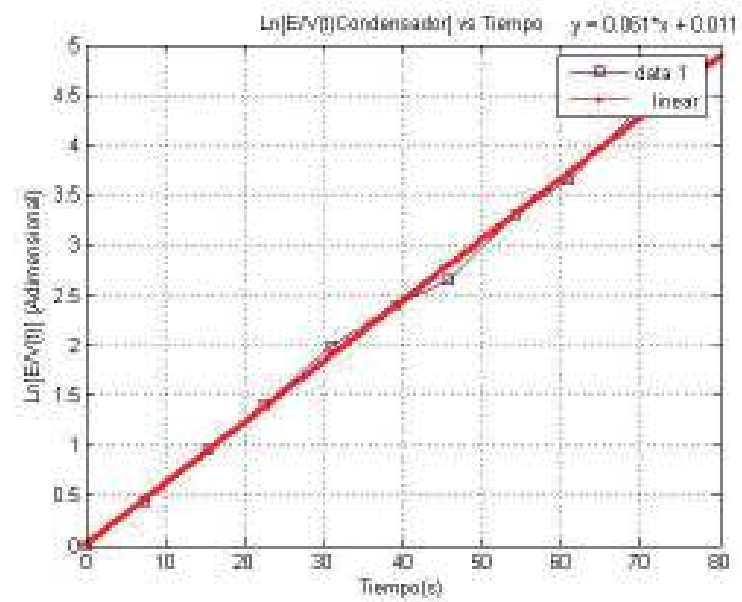

Figura 6. Regresión lineal para la determinación del $\mathrm{RC}$ en el proceso de carga.

Constante de tiempo para la carga aplicando la ecuación (5) es:

$\mathbf{R C}_{\text {exp(1) }}=16.393(\mathrm{~s})$

Constante de tiempo teórico del sistema

$\mathbf{R C}_{\text {teórico }}=15.378(\mathrm{~s})$

Utilizando la Ec (16) 0btenemos: $\boldsymbol{E} \%=$ 6.19\%DESCARGADELCONDENSADOR

TABLA III. DATOS EXPERIMENTALES DEL VOLTAJE Y EL TIEMPO PARA EL PROCESO DE DESCARGA DEL CONDENSADOR.

\begin{tabular}{|c|c|c|c|}
\hline $\begin{array}{c}\mathbf{N}^{\mathbf{0}} \text { de } \\
\text { toma de } \\
\text { datos }\end{array}$ & $\begin{array}{c}\text { Tiempo } \\
(\mathbf{s})\end{array}$ & $\begin{array}{c}\text { Voltaje } \\
\text { Condensador } \\
(\mathbf{V})\end{array}$ & $\begin{array}{c}\text { Voltaje } \\
\text { Resistencia } \\
(\mathbf{V})\end{array}$ \\
\hline 1 & 0,00 & 12,04 & $-12,04$ \\
2 & 7,60 & 7,51 & $-7,51$ \\
3 & 15,50 & 4,50 & $-4,51$ \\
4 & 23,21 & 2,92 & $-2,92$ \\
5 & 33,62 & 1,52 & $-1,50$ \\
6 & 39,73 & 1,02 & $-1,07$ \\
7 & 46,01 & 0,71 & $-0,71$ \\
8 & 53,00 & 0,40 & $-0,40$ \\
9 & 61,01 & 0,26 & $-0,26$ \\
10 & 72,20 & 0,21 & $-0,21$ \\
& & & \\
\hline
\end{tabular}

Utilizando los datos de la tabla 3 construyamos los siguientes gráficos

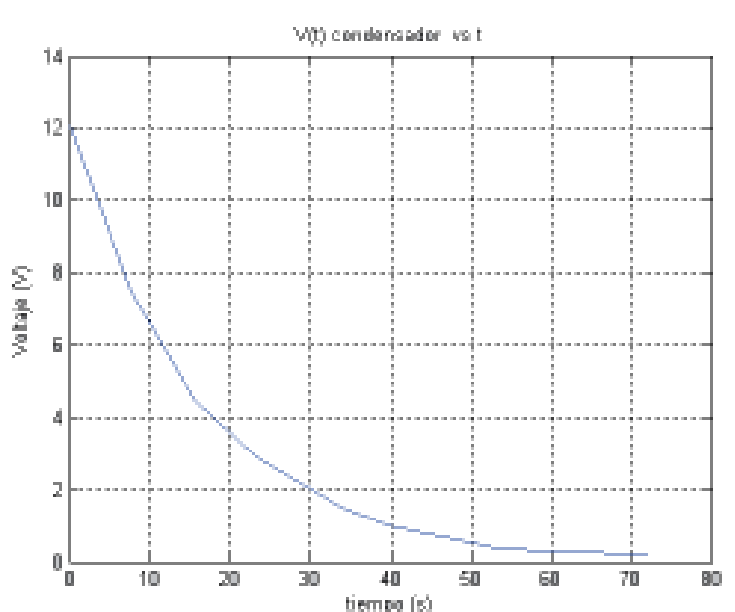

Figura 7. Voltaje del capacitor durante el proceso de descarga en el tiempo

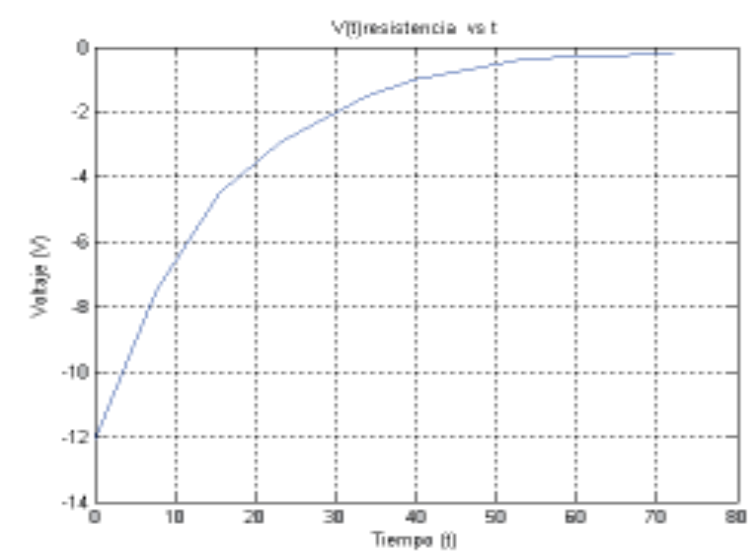

Figura 8. Voltaje de la resistencia durante el proceso descarga en el tiempo

Regresión lineal para la obtención de $\mathrm{RC}_{\text {experimental }}$

en el proceso de descarga del capacitor [10].

$$
\begin{aligned}
& V(t)_{\text {capacitor }}=E e^{-t / R C} \\
& \operatorname{Ln}\left(V(t)_{\text {capacitor }}\right)=\operatorname{Ln}\left(E e^{-t / R C}\right) \\
& \operatorname{Ln}\left(V(t)_{\text {capacitor }}\right)-\operatorname{Ln}(E)=-\left(\frac{1}{R C}\right) t \\
& m t=\operatorname{Ln}\left(\frac{V(t)_{\text {condensado }}}{E}\right)
\end{aligned}
$$


TABLA IV. DATOS EXPERIMENTALES DEL VOLTAJE Y EL TIEMPO PARA EL PROCESO DE DESCARGA DEL CONDENSADOR

\begin{tabular}{|c|c|c|c|c|c|c|}
\hline $\begin{array}{c}\mathbf{N}^{0} \text { de } \\
\text { toma de } \\
\text { datos }\end{array}$ & $\begin{array}{c}\text { Voltaje } \\
(\mathbf{V})\end{array}$ & $\begin{array}{c}\text { Tiempo } \\
(\mathbf{s})\end{array}$ & $\begin{array}{c}\text { FUENTE } \\
\mathbf{E}(\mathbf{V})\end{array}$ & $\begin{array}{c}\text { Ln }(\boldsymbol{E}-\boldsymbol{V} \boldsymbol{i}) \\
(\mathbf{V})\end{array}$ & $\begin{array}{c}\text { Ln }(\boldsymbol{E}) \\
(\mathbf{V})\end{array}$ & $\begin{array}{c}\text { Ln(E-Vi)-In(E) } \\
\text { (adimensional) }\end{array}$ \\
\hline 1 & 12,04 & 0,00 & 12.11 & 2.49 & 2.49 & 0.0000 \\
\hline 2 & 7,51 & 7,60 & 12.11 & 2.02 & 2.49 & -0.4700 \\
\hline 3 & 4,50 & 15,50 & 12.11 & 1.50 & 2.49 & -0.9900 \\
\hline 4 & 2,92 & 23,21 & 12.11 & 1.07 & 2.49 & -1.4224 \\
\hline 5 & 1,52 & 33,62 & 12.11 & 0.42 & 2.49 & -2.0753 \\
\hline 6 & 1,02 & 39,73 & 12.11 & 0.02 & 2.49 & -2.4742 \\
\hline 7 & 0,71 & 46,01 & 12.11 & -0.34 & 2.49 & -2.8365 \\
\hline 8 & 0,40 & 53,00 & 12.11 & -0.92 & 2.49 & -3.4103 \\
\hline 9 & 0,26 & 61,01 & 12.11 & -1.35 & 2.49 & -3.8411 \\
\hline 10 & 0,21 & 72,20 & 12.11 & -1.56 & 2.49 & -4.0547 \\
\hline
\end{tabular}

Pasaremos a graficar los datos de la última columna en función del tiempo para poder hallar la pendiente de la recta determinada por la ecuación (15), la cual es el inverso del $\mathrm{RC}$ tal que estamos buscando. [10]

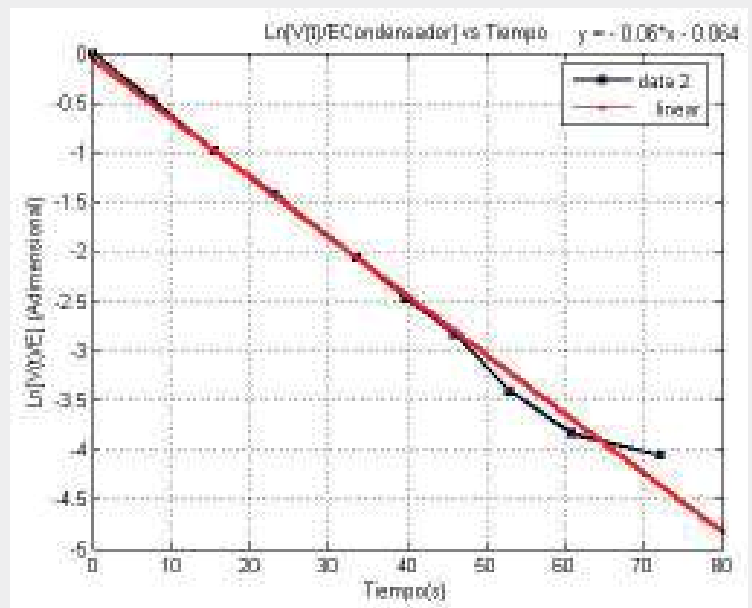

Figura 9. Regresión lineal para la determinación de $\mathrm{RC}$ en el proceso de descarga

Constante de tiempo para la carga aplicando la ecuación (15) es:

$\mathrm{RC}_{\exp (2)}=16.667[\mathrm{~s}]$

Constante de tiempo teórico del sistema

$\mathrm{RC}_{\text {térico }}=15.378[\mathrm{~s}]$

Utilizando la Ec(16) 0btenemos: $\quad \boldsymbol{E} \%=$ $\mathbf{7 . 7 \%}$

\section{CONCLUSIONES}

- Experimentalmente se demostro como los procesos de carga y descarga de un condensador estando conectados en serie con una resistencia y una fuente presentan un comportamiento exponencial.

- Utilizando la segunda ley de Kirchhoff y el cálculo infinitesimal se determinaron las ecuaciones que mostraron el comportamiento de este fenómeno.

- La importancia de la utilización del software Matlab en la construcción de las gráficas, nos permite representar los datos tomados en la práctica y compararlos con la curva de Regresión que el programa realiza.

- Es importante la utilización de los métodos numéricos en la construcción y ajuste de curvas en los datos experimentales, lo que le permite observar al estudiante la relación entre lo teórico y práctico así logra deducir sus propias conclusiones.

- Analizando las gráficas de voltaje vs tiempo en circuitos $\mathrm{RC}$, en el proceso de carga y descarga se pudo confirmar la relación entre las ecuaciones dadas en este fenómeno.

- Habiendo determinado experimentalmente la constante del tiempo RC en dichos procesos, se observó un incremento en la re- 
Eco.Mat

Cúcuta-Colombia

Vol. 6

No. 1

Enero-Diciembre 2015

ISSN 1794-8231

ISSN $2462-8794 \mathrm{Web}$

PP: $34-42$

\section{REFERENCIAS}

[1] Reyes, C. Salcedo, A y Carrillo, F. (2000). Manual de experimentos de Electromagnetismo, Instituto Tecnológico Autónomo de México: México.

[2] Serway, R. (2002). Physics for Scientists and Engineers. Tomo 2. 5ta. ed. México: McGrawhill,

[3] Gutiérrez, C. (2015). Tomado de: http:// carlosurielelectronica.blogspot.com. co/2015/03/blog-post.html

[4] Benítez, M. (2012). La Paz- Bolivia. Tomado de: http://es.scribd.com/doc/172477664/1Electronica-Basica\#scribd.

[5] M. Tipler. (2006). Física para la ciencia y la tecnología. Vol. 2. España: Reverté,

[6] Quintana, P., Villalobos, E. y Cornejo, M. del C. (2005). Numéricos Métodos con aplicaciones en Excel. México: Reverté.

[7] Chapra, S., Canale, R. (2007). Métodos numéricos para ingenieros. Editorial McGraw-Hill. México, D.F. $5^{\text {a }}$.Edición.

[8] Young, H., Freedman, R. (2009). Física Universitaria. Pearson. México.

[9] Ayuso, P. (2011). Ejercicios y problemas del curso de Métodos Numéricos par Ingenieros. California, USA.

[10] Oñate, E. (2011).Métodos numéricos para cálculo y diseño en Ingeniería. España. 